LINEAR AND NON-LINEAR THEORY OF

GENERALIZED FUNCTIONS AND ITS APPLICATIONS

BANACH CENTER PUBLICATIONS, VOLUME 88

INSTITUTE OF MATHEMATICS

POLISH ACADEMY OF SCIENCES

WARSZAWA 2010

\title{
GENERALIZED FUNCTIONS ON ADELES. LINEAR AND NON-LINEAR THEORIES
}

\author{
YAKOV V. RADYNO and YAUHEN M. RADYNA \\ Belarusian State University \\ Nezalezhnastsi av., 4, BY-220030, Minsk, Belarus \\ E-mail: radyno@bsu.by,yauhen.radyna@gmail.com
}

\begin{abstract}
We consider various generalizations of linear homogeneous distributions on adeles and construct a number of algebras of non-linear generalized functions on adeles and totally disconnected groups such as the discrete adeles.
\end{abstract}

1. Introduction. The algebra of adeles over the field of rational numbers $\mathbb{Q}$ was introduced by A. Weil. Invertible adeles, now known as ideles, were introduced by C. Chevalley [4]. Adeles became a powerful analytic tool in number theory [20]; they have applications in representation theory [9] and in modern mathematical physics [6].

There is no interesting topology on the field of rational numbers. However it is possible to embed this field into the canonically associated algebra of adeles having a non-trivial topology and a Haar measure. Thus numerous tools of functional analysis can be applied. Here we summarize some developments in linear and non-linear theory of generalized functions on adeles. See [5] for basics of the non-linear theory on the real axis. An abstract approach to the non-linear theory is presented in $[2,15]$.

\section{The linear theory}

2.1. $p$-adic numbers and adeles. According to Ostrowski's theorem [17] there are countably many non-trivial essentially different valuations on the field $\mathbb{Q}$. One of them is the usual absolute value $|\cdot|_{\infty}=|\cdot|$, and others are $p$-adic valuations $|\cdot|_{p}$ corresponding to the primes $p \in\{2,3,5, \ldots\}$. The $p$-adic valuation is defined by $|x|_{p}=p^{-\gamma}$ for $x=$ $p^{\gamma} \cdot \frac{m}{n} \neq 0$ with integers $m, n$ not divisible by $p$. Notice that $|\cdot|_{p}$ satisfies the strong triangle inequality $|x+y|_{p} \leq \max \left\{|x|_{p},|y|_{p}\right\}$.

2000 Mathematics Subject Classification: Primary 43A70; Secondary 46 F30.

Key words and phrases: adeles, generalized functions, distributions, mnemofunctions.

The paper is in final form and no version of it will be published elsewhere. 
The real field $\mathbb{Q}_{\infty}=\mathbb{R}$ is the completion of $\mathbb{Q}$ with respect to $|\cdot|_{\infty}$. Similarly, the field of $p$-adic numbers $\mathbb{Q}_{p}$ is the completion of $\mathbb{Q}$ with respect to $|\cdot|_{p}$. Each element $x \in \mathbb{Q}_{p}, x \neq 0$, has a unique representation $x=p^{\gamma(x)}\left(x_{0}+x_{1} p+x_{2} p^{2}+\ldots\right)$, where $x_{k} \in\{0,1, \ldots, p-1\}$ are $p$-adic digits, $x_{0} \neq 0$. The field $\mathbb{Q}_{p}$ is locally compact, so there exists a unique shift invariant Haar measure $d_{p} x$ on $\mathbb{Q}_{p}$. We normalize it by $d_{p} x\left(\mathbb{Z}_{p}\right)=1$, where $\mathbb{Z}_{p}=\left\{x:|x|_{p} \leq 1\right\}$ is the ring of $p$-adic integers. For details see $[17,18]$.

Consider finite subsets $S$ of the set $\mathcal{V}=\{\infty, 2,3, \ldots, p, \ldots\}$ with $\infty \in S$ and the rings $\mathbb{A}_{S}$ of sequences given by

$$
\mathbb{A}_{S}=\left\{x=\left(x_{v}\right)_{v \in \mathcal{V}}: \forall v \in S x_{v} \in \mathbb{Q}_{v} \text { and } \forall v \notin S x_{v} \in \mathbb{Z}_{v}\right\}
$$

with pointwise operations. The ring $\mathbb{A}$ of adeles is the union of all such $\mathbb{A}_{S}$. Each $\mathbb{A}_{S}$ has the Tychonoff topology and $\mathbb{A}$ is equipped with the topology of inductive limit.

The field $\mathbb{Q}$ is embedded diagonally into $\mathbb{A}$ by $q \mapsto(q, q, q, \ldots)$. The embedding is well-defined since $|q|_{p}=1$ for all but a finite number of $p$ 's. Thus $\mathbb{A}$ is an algebra over $\mathbb{Q}$ and the image of the embedding forms a discrete co-compact subgroup of $\mathbb{A}$ with respect to addition.

The multiplicative group $\mathbb{A}^{\times}$of ideles has a topology induced by the embedding $\lambda \mapsto\left(\lambda, \lambda^{-1}\right)$ into $\mathbb{A} \times \mathbb{A}$. For each idele $\lambda$ one defines its modulus

$$
|\lambda|=\prod_{v}\left|x_{v}\right|_{v} \in(0,+\infty) \quad \text { (the first adelic formula states that }|q|=1 \text { for } q \in \mathbb{Q}^{\times} \text {). }
$$

One has $\mathbb{A}=\mathbb{R} \times \mathbb{A}_{0}$, where $\mathbb{A}_{0}$ is the discrete adele group. The groups $\mathbb{A}, \mathbb{A}_{0}$ and $\mathbb{A}^{\times}$ are locally compact. For details we refer to $[9,20]$.

Bruhat-Schwartz distributions. The Bruhat-Schwartz space $\mathcal{S}(\mathbb{A})$ of test functions on adeles consists of finite linear combinations of cylindrical functions $\varphi(\lambda)=\prod_{\nu} \varphi_{\nu}\left(\lambda_{\nu}\right)$, where $\varphi_{\infty} \in \mathcal{S}(\mathbb{R}), \varphi_{p} \in \mathcal{S}\left(\mathbb{Q}_{p}\right)$ and all but finitely many of $\varphi_{p}$ are equal to the characteristic function of $\mathbb{Z}_{p}$. Recall that $\mathcal{S}\left(\mathbb{Q}_{p}\right)$ consists of locally constant and compactly supported functions on $\mathbb{Q}_{p}$. The Bruhat-Schwartz space $\mathcal{S}\left(\mathbb{A}_{0}\right)$ also consists of locally constant compactly supported functions and can be represented as an inductive limit of compactly supported spaces; $\mathcal{S}\left(\mathbb{A}_{0}\right)$ carries the strongest locally-convex topology, so $\mathcal{S}(\mathbb{A})=\mathcal{S}(\mathbb{R}) \otimes \mathcal{S}\left(\mathbb{A}_{0}\right)$. Elements of the dual spaces $\mathcal{S}^{\prime}(\mathbb{A})$ and $\mathcal{S}^{\prime}\left(\mathbb{A}_{0}\right)$ are called BruhatSchwartz distributions [3].

Pontryagin duality and Fourier transform. The fractional part of $x \in \mathbb{Q}_{p}$ is the number

$$
\{x\}_{p}=\left\{\begin{array}{l}
0, \quad \text { if } \gamma(x) \geq 0 \text { or } x=0, \\
p^{\gamma}\left(x_{0}+\ldots+x_{|\gamma|-1} p^{|\gamma|-1}\right), \text { if } \gamma(x)<0
\end{array} \in \mathbb{Q} .\right.
$$

Consider the map $\chi: \mathbb{A} \rightarrow \mathbb{C}$ given by

$$
\chi(x)=\exp \left(2 \pi i\left(-\left\{x_{\infty}\right\}+\left\{x_{2}\right\}_{2}+\left\{x_{3}\right\}_{3}+\ldots+\left\{x_{p}\right\}_{p}+\ldots\right)\right) .
$$

One checks [9] that $\chi$ is a character (i.e. $\chi(x+y)=\chi(x) \chi(y),|\chi(x)|=1$, see [11] for a general theory). The Pontryagin dual group $\widehat{\mathbb{A}}$ can be identified with $\mathbb{A}$ by the homeomorphism $s \mapsto \chi_{s}$ given by $\chi_{s}(t)=\chi(s t)$.

The Fourier transform $\mathcal{F}: \mathcal{S}(\mathbb{A}) \rightarrow \mathcal{S}(\mathbb{A})$ acts on cylindrical functions by $\mathcal{F}: \otimes_{v} \varphi_{v} \mapsto$ $\otimes_{v}\left(\mathcal{F}_{v} \varphi_{v}\right)$. The Fourier transform on $\mathcal{S}^{\prime}\left(\mathbb{A}_{0}\right)$ is defined by duality $\langle\mathcal{F} u, \varphi\rangle=\langle u, \mathcal{F} \varphi\rangle$. The 
Fourier transform is a linear isomorphism both on $\mathcal{S}(\mathbb{A})$ and on $\mathcal{S}^{\prime}(\mathbb{A})$. A convolution is also defined on $\mathcal{S}(\mathbb{A})$. It is connected with the Fourier transform in the usual way.

2.2. Adelic distributions $\Delta_{g, \theta}$ and a functional equation. Let $\mathbb{R}_{+}=(0,+\infty)$. Consider a locally integrable tempered complex-valued function $g \in L_{1}^{\text {loc }}\left(\mathbb{R}_{+}\right) \cap \mathcal{S}^{\prime}\left(\mathbb{R}_{+}\right)$. Let $\theta$ be a character of the group $\mathbb{A}^{\times} / \mathbb{Q}^{\times} ; \theta$ can be seen as a character of $\mathbb{A}^{\times}$satisfying $\theta(q)=1$ for $q \in \mathbb{Q}^{\times}$. We define the Bruhat-Schwartz distribution $\Delta_{g, \theta}$ as the integral over ideles with respect to the idelic Haar measure

$$
\Delta_{g, \theta}(\varphi)=\int_{\mathbb{A}^{\times}} \varphi(\lambda) \theta(\lambda) g(|\lambda|) d^{*} \lambda, \quad \varphi \in \mathcal{S}(\mathbb{A})
$$

The case $g(t)=t^{s}$ gives the famous Tate distributions (also known as homogeneous distributions $[9,10])$. In general, the integral above does not converge and one needs a regularization.

Lemma 2.1. If $\operatorname{supp}(g) \subset[T,+\infty)$ for some $T>0$ then $\Delta_{g, \theta}$ is well-defined.

This lemma and the Poisson-Tate summation formula

$$
\sum_{q \in \mathbb{Q}} \varphi(q \lambda)=\frac{1}{|\lambda|} \sum_{q \in \mathbb{Q}} \tilde{\varphi}\left(\frac{q}{\lambda}\right), \quad \lambda \in \mathbb{A}^{\times}, \varphi \in \mathcal{S}(\mathbb{A}),
$$

allow us to write the following regularization formula [14]

$$
\Delta_{g, \theta}(\varphi)=\Delta_{g, \theta}^{+}(\varphi)+\Delta_{g^{\tau}, \bar{\theta}}^{+}(\mathcal{F} \varphi)+\varepsilon_{\theta}(\mathcal{F} \varphi)(0) \int_{(0,1]} \frac{g(t)}{t} d^{*} t-\varepsilon_{\theta} \varphi(0) \int_{(0,1]} g(t) d^{*} t .
$$

Here $\Delta_{g, \theta}^{+}$denotes the well-defined distribution $\Delta_{f, \theta}$, where $f(t)=I_{[1,+\infty)} g(t), I_{[1,+\infty)}$ is the characteristic function of the interval, $g^{\tau}(t)=t \cdot g\left(t^{-1}\right), \varepsilon_{\theta}=1$ if $\theta \equiv 1$, and $\varepsilon_{\theta}=0$ otherwise. To complete the regularization in case $\theta \equiv 1$ one should assume that certain values have been assigned to $\int_{(0,1]} \frac{g(t)}{t} d^{*} t$ and $\int_{(0,1]} g(t) d^{*} t$. Assuming also that certain values have been assigned to $\int_{(0,1]} g^{\tau}(t) d^{*} t$ and $\int_{(0,1]} \frac{g^{\tau}(t)}{t} d^{*} t$ we can write the Fourier image of $\Delta_{g, \theta}$ in the following form

$$
\left(\mathcal{F} \Delta_{g, \theta}\right)(\varphi)=\Delta_{g^{\tau}, \bar{\theta}}(\varphi)+\varepsilon_{\theta} \varphi(0) \int_{(0,+\infty)} \frac{g(t)}{t} d^{*} t-\varepsilon_{\theta}(\mathcal{F} \varphi)(0) \int_{(0,+\infty)} g(t) d^{*} t .
$$

Using the regularization technique as above we deduce

THEOREM 2.2. Any Bruhat-Schwartz function on the group of idele classes can be regarded as an adelic distribution. Precisely, there is a continuous embedding $\mathcal{S}\left(\mathbb{A}^{\times} / \mathbb{Q}^{\times}\right) \rightarrow$ $\mathcal{S}^{\prime}(\mathbb{A})$.

Let $\Delta(g(t))$ denote a distribution $\Delta_{g, \theta}$, where $\theta \equiv 1$. Let $\delta$ denote the Dirac delta, and let 1 denote the distribution in $\mathcal{S}^{\prime}(\mathbb{A})$ corresponding to the constant 1 . For the characteristic function of a segment, $g(t)=I_{[a, b]}(t), a, b>0$, one has

$$
\mathcal{F} \Delta\left(I_{[a, b]}\right)=\Delta\left(t \cdot I_{[1 / b, 1 / a]}\right)+\delta \cdot(\log b-\log a)-\mathbf{1} \cdot \varphi(0)(b-a) .
$$

For $g(t)=t^{s} \cdot h(t)$ depending on $s \in \mathbb{C}$ our functional equation simplifies to

$$
\mathcal{F} \Delta\left(t^{s} \cdot h(t)\right)=\Delta\left(t^{1-s} \cdot h\left(t^{-1}\right)\right)+\delta \cdot(M h)(s-1)-\mathbf{1} \cdot(M h)(s),
$$


where $(M h)(s)$ denotes the Mellin transform of $h$ at $s$. In this case analytic continuation with respect to $s$ can be applied. For example,

$$
\mathcal{F} \Delta\left(\frac{t^{s}}{a+t^{r}}\right)=\Delta\left(\frac{t^{1-s}}{a+t^{-r}}\right)+\delta \cdot \frac{\pi a^{\frac{(s-1)}{r}-1}}{r \sin \frac{\pi(s-1)}{r}}-\mathbf{1} \cdot \frac{\pi a^{\frac{s}{r}-1}}{r \sin \frac{\pi s}{r}}, a>0, s \notin r \mathbb{Z} .
$$

2.3. A multi-index generalization of Tate distribution. Let $\xi=\left(\xi_{2}, \xi_{3}, \ldots, \xi_{p}, \ldots\right)$ $\in \mathbb{A}_{0}$ and let $\alpha=\left(\alpha_{2}, \alpha_{3}, \ldots, \alpha_{p}, \ldots\right) \in \mathbb{R}^{\infty}$ be an infinite multi-index. The formal expression $|\xi|^{\alpha}=\prod_{p}\left|\xi_{p}\right|_{p}^{\alpha_{p}}$ generalizes homogeneous distributions on the group of discrete adeles $[7,19]$.

THEOREM 2.3. If $\lim _{p \rightarrow \infty} \alpha_{p} \ln p=0$, then $|\xi|^{\alpha}$ represents a function finite almost everywhere with respect to the Haar measure. It belongs to $L_{q}^{\text {loc }}\left(\mathbb{A}_{0}\right), 1 \leq q<+\infty$, if $\alpha_{p}>-1 / q$ for all $p$. It does not belong to $L_{q}\left(\mathbb{A}_{0}\right)$ for any $1 \leq q<+\infty$.

COROLlaRY 2.4. If $\lim _{p \rightarrow \infty} \alpha_{p} \ln p=0$ and $\alpha_{p}>-1$ for all $p$, then $|\xi|^{\alpha}$ is finite almost everywhere. In particular, $|\xi|^{\alpha} \in \mathcal{S}^{\prime}\left(\mathbb{A}_{0}\right)$.

Assume that $\lim _{p \rightarrow \infty} \alpha_{p} \ln p=0$ and $\alpha_{p}>-1 / 2$ for all $p$. The Vladimirov operator $V^{\alpha}$ is a pseudo-differential operator with the symbol $|\xi|^{\alpha}$ :

$$
\left(V^{\alpha} \varphi\right)(x)=\int_{A}|\xi|^{\alpha} \varphi(\xi) \chi_{0}(-\xi x) d \xi, \quad \varphi(x) \in \mathcal{S}\left(\mathbb{A}_{0}\right) .
$$

Here $\chi_{0}$ is a basic character for discrete adeles similar to $\chi$ in section 2.1.

THEOREM 2.5. The operator $V^{\alpha}$ with the domain $\mathcal{S}\left(\mathbb{A}_{0}\right)$ is essentially self-adjoint. Its closure (let us denote it by $V^{\alpha}$ again) with the domain

$$
D\left(V^{\alpha}\right)=\left\{\varphi \in L_{2}\left(\mathbb{A}_{0}\right):|\xi|^{\alpha} \varphi(\xi) \in L_{2}\left(\mathbb{A}_{0}\right)\right\}
$$

is a self-adjoint operator with spectrum $\sigma\left(V_{\alpha}\right)=[0,+\infty)$.

\section{Non-linear generalized functions on adeles}

3.1. The case of a totally disconnected self-dual group. Now we pass to the nonlinear theory of adelic generalized functions. First we describe a more general approach to construct algebras of non-linear generalized functions (mnemofunctions) than in $[13,14]$. It applies to any totally disconnected self-dual group $G$ such as $\mathbb{Q}_{p}$ or $\mathbb{A}_{0}$, see $[1,12]$ for the $p$-adic theory. Let us fix some isomorphism $\alpha: G \rightarrow \widehat{G} \cong G$ and consider the self-dual Haar measure $m$ on $G$.

Everywhere below $H$ denotes an open compact subgroup of $G$. Its annihilator $H^{\perp}:=$ $\{g \in G: \forall h \in H\langle\alpha(g), h\rangle=1\} \subset G$ is also an open compact subgroup.

We call $H$ a small subgroup if $H \subset H^{\perp}$. Let $\mathcal{H}$ denote the family of all small subgroups (subgroup $H_{0} \cap H_{0}^{\perp}$ is small for any open compact $H_{0}$, so $\mathcal{H}$ is not empty). The family $\mathcal{H}$ is ordered by inverse inclusion. One checks that $\mathcal{H}$ forms a neighborhood filter at zero. Everywhere below $H$ is small.

The Bruhat-Schwartz space $\mathcal{S} \equiv \mathcal{S}(G)$ consists of all locally constant compactly supported functions $\varphi$ (i.e. for some $H$ one has $(x-y) \in H \Rightarrow \varphi(x)=\varphi(y)$ and $\left.\operatorname{supp} \varphi \subset H^{\perp}\right)$. 
Consider the following algebra $\mathcal{G}_{M}$ of nets indexed by $\mathcal{H}$ and the ideal $\mathcal{N} \subset \mathcal{G}_{M}$ :

$$
\begin{gathered}
\mathcal{G}_{M} \stackrel{\text { def }}{=}\left\{g=\left(g_{H}\right)_{H \in \mathcal{H}}: g_{H} \in \mathcal{S}\right\}=\mathcal{S}^{\mathcal{H}}, \\
\mathcal{N} \stackrel{\text { def }}{=}\left\{g \in \mathcal{G}_{M}: \exists H_{0} \quad \forall H \succeq H_{0} \quad g_{H} \equiv 0\right\} .
\end{gathered}
$$

We call the factor algebra $\mathcal{G}=\mathcal{G}_{M} / \mathcal{N}$ the algebra of Egorov type mnemofunctions on $G$ (for the original construction of Egorov see [8]). As usual, we say that $g, h \in \mathcal{G}_{M}$ are associated if $\left(g_{H}-f_{H}\right) \rightarrow 0$ in $\mathcal{S}^{\prime}$. This definition extends to mnemofunctions in $\mathcal{G}$.

Consider the canonical $\delta$-net $\left(\delta_{H}\right)_{H \in \mathcal{H}}$ and the canonical 1-net $\left(\mathbf{1}_{H}\right)_{H \in \mathcal{H}}$ defined by

$$
\delta_{H}=m(H)^{-1} \cdot I_{H} \in \mathcal{S}, \quad \mathbf{1}_{H}=\mathcal{F} \delta_{H}=I_{H^{\perp}} \in \mathcal{S},
$$

where $m(H)$ is the Haar measure of $H$ and $\mathcal{F} \delta_{H}$ is the Fourier image of $\delta_{H}$.

We define the regularization operator $R: \mathcal{S}^{\prime} \rightarrow \mathcal{G}_{M}$ and the embedding $\tau: \mathcal{S}^{\prime} \rightarrow \mathcal{G}$ of distribution space into our mnemofunctional algebra by

$$
\begin{gathered}
R: \mathcal{S}^{\prime} \rightarrow \mathcal{G}_{M}: u \mapsto R(u) \stackrel{\text { def }}{=}\left(\left(u * \delta_{H}\right) \cdot \mathbf{1}_{H}\right)_{H \in \mathcal{H}}, \\
\tau: \mathcal{S}^{\prime} \rightarrow \mathcal{G}: u \mapsto[R(u)] \stackrel{\text { def }}{=} R(u)+\mathcal{N} .
\end{gathered}
$$

Lemma 3.1. For any $u \in \mathcal{S}^{\prime}$ its regularization $R(u)$ converges to $u$ in $\mathcal{S}^{\prime}$.

In particular, $\delta_{H}=R(\delta)_{H}$ converges to the Dirac delta-function, and $\mathbf{1}_{H}=R(\mathbf{1})_{H}$ converges to the constant $\mathbf{1} \in \mathcal{S}^{\prime}$. Lemma 3.1 implies injectivity of $R$ and $\tau$.

THEOREM 3.2. The restriction $\tau: \mathcal{S}^{\prime} \rightarrow \mathcal{G}$ onto the subalgebra $\mathcal{S} \subset \mathcal{S}^{\prime}$ coincides with the canonical embedding $\tau^{\prime}: \mathcal{S} \rightarrow \mathcal{G}: a \mapsto\left[(a)_{H \in \mathcal{H}}\right]$. Thus $\tau$ yields an injective homomorphism of the algebra $\mathcal{S}$.

The relation between the embedding and the multiplication is described by TheOrem 3.3. $1^{\circ}$ For any $a \in \mathcal{S}, u \in \mathcal{S}^{\prime}$ the product $(a u)$ belongs to $\mathcal{S}^{\prime}$ and $\tau(a u)=$ $\tau(a) \tau(u)$. In other words, $\tau$ preserves the structure of $\mathcal{S}$-module on $\mathcal{S}^{\prime} .2^{\circ}$ For any continuous $u, v \in C(G)$ their pointwise product (uv) belongs to $C(G)$ and the mnemofunctions $\tau(u) \tau(v), \tau(u v)$ are associated.

Note that the analogue of $1^{\circ}$ in Theorem 3.3 does not hold in the real case [16].

We define the Fourier transform on $\mathcal{G}$ by

$$
\mathcal{F}: \mathcal{G} \rightarrow \mathcal{G}:\left[\left(g_{H}\right)_{H \in \mathcal{H}}\right] \mapsto\left[\left(\mathcal{F} g_{H}\right)_{H \in \mathcal{H}}\right]
$$

THEOREM 3.4. The embedding $\tau$ intertwines the Fourier transform of distributions and the Fourier transform of mnemofunctions, i.e. for all $u \in \mathcal{S}^{\prime}$ one has the equality

$$
\mathcal{F}(\tau(u))=\tau(\mathcal{F} u)
$$

The above is again in contrast with the real case, because we have an exact equality here, but not the property of being associated.

The problem of defining a convolution on $\mathcal{S}^{\prime}$, i.e. a bilinear map $*: \mathcal{S}^{\prime} \times \mathcal{S}^{\prime} \rightarrow \mathcal{S}^{\prime}$ that would be a reasonable continuation of the convolution on $\mathcal{S}$, is equivalent to the problem of multiplication. This is easily seen after taking the Fourier transform. 
We define the convolution on $\mathcal{G}$ by the formula

$$
*: \mathcal{G} \times \mathcal{G} \rightarrow \mathcal{G}:\left(\left[\left(f_{H}\right)_{H \in \mathcal{H}}\right],\left[\left(g_{H}\right)_{H \in \mathcal{H}}\right]\right) \mapsto\left[\left(f_{H} * g_{H}\right)_{H \in \mathcal{H}}\right] .
$$

The definition is correct, since $\mathcal{N} * \mathcal{N} \subset \mathcal{N}$ and $\mathcal{N} * a \subset \mathcal{N}$ for all $a \in \mathcal{G}_{M}$.

THEOREM 3.5. $1^{\circ}$ The restriction of $\tau$ onto $\mathcal{S}$ is a morphism of convolution algebras, i.e.

$$
\tau(a * b)=\tau(a) * \tau(b), \quad a, b \in \mathcal{S} .
$$

$2^{\circ}$ The convolution algebra $(\mathcal{G}, *,+)$ is isomorphic to $(\mathcal{G}, \times,+)$. The isomorphism is the Fourier transform.

The developed technique is easily applied in the $p$-adic case $[12,14]$.

Lemma 3.6. Small subgroups $H \subset \mathbb{Q}_{p}$ have the form $H=p^{n} \mathbb{Z}_{p}, n \in\{0,1,2, \ldots\}$. The ordered set $\mathcal{H}$ is isomorphic to $\{0,1,2, \ldots\}$ with the usual order.

EXAMPLE 3.7. The $p$-adic homogeneous distribution $\Delta_{s}=|x|_{p}^{s-1}, s \notin \frac{2 \pi i}{\log p} \mathbb{Z}$, is represented by the following mnemofunction

$$
R\left(\Delta_{s}\right)_{n}=|x|_{p}^{s-1} \cdot I_{B_{n} \backslash B_{-n}}+\left(1-p^{-1}\right) \frac{p^{(1-s) n}}{1-p^{-s}} \cdot I_{B_{-n}} .
$$

Here $B_{n}=B\left[0, p^{n}\right]$ is a closed ball.

EXAMPLE 3.8. The Vladimirov finite part $\mathcal{P} \frac{1}{|x|_{p}}$ is represented by

$$
R\left(\mathcal{P} \frac{1}{|x|_{p}}\right)_{n}=|x|_{p}^{-1} \cdot I_{B_{n} \backslash B_{-n}}(x)-\left(1-p^{-1}\right) n p^{n} \cdot I_{B_{-n}}(x) .
$$

\subsection{Mnemofunctions on discrete adeles $\mathbb{A}_{0}$}

Lemma 3.9. Small subgroups $H \subset \mathbb{A}_{0}$ have the form $H=\prod_{p} p^{\alpha_{p}} \mathbb{Z}_{p}$, where $\alpha_{p} \in \mathbb{Z}$, $\alpha_{p} \geq 0$, and $\alpha_{p}=0$ for almost all $p$.

The ordered set $\mathcal{H}$ can be described by non-negative rational divisors $\alpha \in \operatorname{Div}^{+} \mathbb{Q}$. Divisors are elements of the free Abelian group Div $\mathbb{Q}$ generated by all non-Archimedean completions of $\mathbb{Q}, \alpha \equiv \sum_{p} \alpha_{p}(p)$, where $\alpha_{p} \in \mathbb{Z}$ and $\alpha_{p}=0$ for almost all $p$. There is a natural order $\succeq$ on Div $\mathbb{Q}$, namely $\alpha \succeq \beta \stackrel{\text { def }}{\Leftrightarrow} \alpha_{\nu} \geq \beta_{\nu}$ for all $\nu$. We call a divisor $\alpha$ non-negative if $\alpha \succeq 0$ with respect to it. There is an isomorphism of ordered sets between divisors and open compact subgroups

$$
\operatorname{Div} \mathbb{Q} \ni \alpha \equiv \sum_{p} \alpha_{p}(p) \leftrightarrow \prod_{p} p^{\alpha_{p}} \mathbb{Z}_{p} \equiv H_{\alpha} .
$$

The following lemma is important for computations.

Lemma 3.10. For any divisor $\alpha \in \operatorname{Div} \mathbb{Q}$ we have $H_{\alpha}^{\perp}=H_{-\alpha}$.

Thus $\mathcal{H} \cong \operatorname{Div}^{+} \mathbb{Q}$. Note that $\operatorname{Div}^{+} \mathbb{Q}$ is also isomorphic to the set of positive integers with the divisibility order. The isomorphism is given by $\alpha \leftrightarrow \prod_{p} p^{\alpha_{p}}$.

Example 3.11. Let $s \in \mathbb{C}, \operatorname{Re} s>1$. Consider the non-Archimedean part $\Delta_{s}^{(0)}$ of the Tate distribution $\Delta_{s, \theta}$ in the case $\theta \equiv 1$ given by 


$$
\Delta_{s}^{(0)}=\bigotimes_{p} \frac{\left|x_{p}\right|_{p}^{s-1}}{1-p^{-1}}
$$

Identifying $\mathcal{H}$ with the positive integers we check that $[13,14]$

$$
R_{n}\left(\Delta_{s}^{(0)}\right)=\frac{\zeta(s)}{n^{s-1}} \bigotimes_{p}\left(I_{n \mathbb{Z}_{p}}\left(x_{p}\right)+\frac{1-p^{-s}}{1-p^{-1}} \cdot\left|\frac{x_{p}}{n}\right|_{p}^{s-1} I_{n^{-1} \mathbb{Z}_{p} \backslash n \mathbb{Z}_{p}\left(x_{p}\right)}\right) .
$$

Here $\zeta(s)$ is the Riemann zeta-function.

3.3. Adelic mnemofunctions. The group $\mathbb{A}$ of adeles is not totally disconnected, so the construction of subsection 3.1 cannot be applied directly. Instead we will use the group of Arakelov divisors of the rational field $\operatorname{Div}_{A} \mathbb{Q} \stackrel{\text { def }}{=} \mathbb{R} \times \operatorname{Div} \mathbb{Q}$. Its elements are formal sums over all completions of the rational field of the form

$$
\alpha=\alpha_{\infty}(\infty)+\sum_{p} \alpha_{p}(p), \quad \alpha_{\infty} \in \mathbb{R}, \alpha_{p} \in \mathbb{Z}, \alpha_{p}=0 \text { for almost all } p .
$$

There is a natural order on $\operatorname{Div}_{A} \mathbb{Q}$, namely $\alpha \succeq \beta \stackrel{\text { def }}{\Leftrightarrow} \alpha_{\nu} \geq \beta_{\nu}$ for all $\nu$. We denote the set of all non-negative $\alpha \succeq 0$ by $\operatorname{Div}_{A}^{+} \mathbb{Q}$.

The algebra $\mathcal{G}_{M}$ in our case is simply $\mathcal{G}_{M}=\mathcal{S}^{\operatorname{Div}_{A}^{+} \mathbb{Q}}$ and the ideal $\mathcal{N}$ is defined by

$$
\mathcal{N} \stackrel{\text { def }}{=}\left\{g \in \mathcal{G}_{M}: \exists \alpha_{0} \quad \forall \alpha \succeq \alpha_{0} \quad g_{\alpha} \equiv 0\right\} \subset \mathcal{G}_{M} .
$$

There are no open compact subgroups attached to divisors. Instead we use an auxiliary function as in the real case [16]. Let $\psi: \mathbb{R} \rightarrow[0,1]$ be an infinitely differentiable compactly supported function equal to 1 in some neighborhood of zero. Let $\left(\mathbf{1}_{\psi, \alpha}\right)_{\alpha \in \operatorname{Div}_{A}^{+} \mathbb{Q}}$ be a $\mathbf{1}$-net given by

$$
\mathbf{1}_{\psi, \alpha}=\mathbf{1}_{\psi, \Lambda}^{(\infty)} \otimes \bigotimes_{p} \mathbf{1}_{\alpha_{p}}^{(p)}=\psi\left(\frac{x_{\infty}}{\Lambda}\right) \otimes \bigotimes_{p} I_{\mathbb{Z}_{p}}\left(p^{\alpha_{p}} \cdot x_{p}\right), \quad \Lambda=\exp \left(\alpha_{\infty}\right) .
$$

The Fourier images $\delta_{\psi, \alpha} \stackrel{\text { def }}{=} \mathcal{F} \mathbf{1}_{\psi, \alpha}$ given by

$$
\delta_{\psi, \alpha}=\delta_{\psi, \Lambda}^{(\infty)} \otimes \bigotimes_{p} \delta_{\alpha_{p}}^{(p)}=\left(\Lambda \widehat{\psi}\left(\Lambda \cdot x_{\infty}\right)\right) \otimes \bigotimes_{p}\left(p^{\alpha_{p}} \cdot I_{\mathbb{Z}_{p}}\left(p^{-\alpha_{p}} \cdot x_{p}\right)\right)
$$

form a $\delta$-net.

The corresponding regularization operator $R_{\psi}$ and the embedding $\tau_{\psi}$ of the space $\mathcal{S}^{\prime}(\mathbb{A})$ of distributions into the mnemofunction algebra $\mathcal{G}$ are given by

$$
\begin{gathered}
R_{\psi}: \mathcal{S}^{\prime}(\mathbb{A}) \rightarrow \mathcal{G}_{M}: u \mapsto\left(\left(u * \delta_{\psi, \alpha}\right) \cdot \mathbf{1}_{\psi, \alpha}\right)_{\alpha \in \operatorname{Div}_{A}^{+} \mathbb{Q}}, \\
\tau_{\psi}: \mathcal{S}^{\prime}(\mathbb{A}) \rightarrow \mathcal{G}: u \mapsto[R(u)] \stackrel{\text { def }}{=} R(u)+\mathcal{N} .
\end{gathered}
$$

One can prove analogous results concerning convergence, embedding, multiplication and Fourier transform to the results of subsection 3.1. The only difference is that all equalities in the theorems therein should be replaced with the relation of being associated.

Acknowledgements. The authors thank the Organizing Committee of the GF'07 conference for kind invitation and support. 


\section{References}

[1] S. Albeverio, A. Yu. Khrennikov and V. M. Shelkovich, Non-linear singular problems in p-adic analysis: associative algebras of p-adic distributions, Izv. Math. 69 (2005), 221-263.

[2] A. B. Antonevich and Ya. V. Radyno, On general methods of constructing algebras of generalized functions, Dokl. Akad. Nauk SSSR 318 (1991), 267-270 (in Russian).

[3] F. Bruhat, Distributions sur un groupe localement compact et applications à l'étude des représentations des groupes p-adiques, Bull. Soc. Math. France 89 (1961), 43-75.

[4] C. Chevalley, La théorie du corps de classes, Ann. Math. 41 (1940), 394-418.

[5] J.-F. Colombeau, Elementary Introduction to New Generalized Functions, North-Holland, Amsterdam, 1985.

[6] B. Dragovich, On generalized functions in adelic quantum mechanics, Integral Transforms Spec. Func. 6 (1998), 197-203.

[7] B. Dragovich, Ya. Radyno and A. Khrennikov, Distributions on adeles, J. Math. Sci. 142 (2007), 2105-2112.

[8] Yu. V. Egorov, On the theory of generalized functions, Uspekhi Mat. Nauk 45 (1990), no. 5, 3-40 (in Russian).

[9] I. M. Gelfand, M. I. Graev and I. I. Piatetski-Shapiro, Representation Theory and Automorphic Functions, Saunders, Philadelphia, 1969.

[10] S. Haran, Riesz potentials and explicit sums in arithmetics, Invent. Math. 101 (1990), 697-703.

[11] S. Morris, Pontryagin Duality and the Structure of Locally Compact Abelian Groups, Cambridge University Press, 1977.

[12] Ya. M. Radyna, Mnemofunctions of Egorov type on $\mathbb{Q}_{p}$, Dokl. Nats. Akad. Nauk Belarusi 47 (2003), no. 4, 10-13 (in Russian).

[13] Ya. M. Radyna, Adelic mnemofunctions and Fourier transform, Dokl. Nats. Akad. Nauk Belarusi, 48 (2004), no. 4, 10-14 (in Russian).

[14] Ya. M. Radyna and Ya. V. Radyno, Distributions and mnemofunctions on adeles. Fourier transform, Proc. Steklov Inst. Math. 245 (2004), 215-227.

[15] Ya. V. Radyno, Extensions of algebras, mnemofunctions and their applications, in: Nonlinear Theory of Generalized Functions. Chapman\&Hall/CRC, 1997, 209-218.

[16] Ya. V. Radyno, N. Ph. Thanh and R. Sabra, The Fourier transform in the algebra of new generalized functions, Dokl. Ross. Akad. Nauk 327 (1992), 20-24 (in Russian).

[17] W. H. Schikhof, Ultrametric Calculus. An Introduction to p-adic Analysis, Cambridge University Press, 1984.

[18] V. S. Vladimirov, I. V. Volovich and E. I. Zelenov, p-adic Analysis and Mathematical Physics, World Scientific, Singapore, 1994.

[19] I. V. Volovich, Ya. V. Radyno and A. Yu. Khrennikov, Generalized functions and Vladimirov operator on group of finite adeles, Infinite Dimensional Analysis, Quantum Probability and Related Topics 6 (2003), 34-59.

[20] A. Weil, Basic Number Theory, Springer-Verlag, New York, 1995. 